\title{
Branching Space-Times and Parallel Processing
} (draft version; the final version appeared in H. Andersen, D. Dieks, W.J. Gonzalez,

T. Uebel, G. Wheeler (eds.), New Challenges to Philosophy of Science, The

Philosophy of Science in a European Perspective Volume 4, 2013, pp. 135-148)

\section{Leszek Wroński*}

February 24, 2012

\begin{abstract}
There is a remarkable similarity between some mathematical objects used in the Branching Space-Times framework and those appearing in computer science in the fields of event structures for concurrent processing and Chu spaces. This paper introduces the similarities and formulates a few open questions for further research, hoping that both BST theorists and computer scientists can benefit from the project.
\end{abstract}

\section{Introduction}

The goal of this short paper is to put forward a few open questions regarding the connections between two areas, one mainly of interest to philosophers, the other to computer scientists: the theory of Branching Space-Times (BST) and the field of modelling parallel processing. The hope is that establishing these connections will eventually help to solve some fundamental technical difficulties of the BST approach, while allowing some types of structures from the realm of computer science to have a spatiotemporal representation.

Why should a theory which, judging by its name, concerns branching spacetimes, be in any way connected to parallel processing? Consider first the well known theory of Branching Time (BT): any BT structure can be viewed as modelling the way a certain indeterministic process could go. It would seem that a theory which allows modelling of bundles of (possibly) indeterministic processes is just a step away, requiring only the modification of the representation of maximal possible courses of events: they should no longer be linear, but should have a spatial dimension. This, however, would still not be enough to capture the idea of independent choices (or indeterministic events), and thus the relationship between BT and BST is a bit more complicated. In the next section

${ }^{*}$ This paper stems from a joint project with Thomas Müller (Universiteit Utrecht), who told me of the idea, triggered by a remark by Hu Liu, of connecting the Branching Space-Times theory to the approaches to parallel processing found in computer science. 
we introduce the two approaches and state the first open problem about BST. In the two sections that follow we sketch two approaches to parallel processing in computer science: that of "event structures" (ES) and that of Chu spaces. The results we have in mind concern the methods of generating structures of a given type given a structure of another type so that some important "structural" infomation is preserved; for example, how to construct a Chu space given a BST model and vice versa. A method for generating an event structure on the basis of a Chu Space is sketched at the end of section 4.1.

\section{Branching Time and Branching Space-Times}

\subsection{Branching Time (BT) structures}

Definition 1 A $B T$ structure is a pair $\langle W, \leqslant\rangle$ such that:

- $W \neq \emptyset$

- $\leqslant$ is a partial order on $\mathrm{W}$;

- $\leqslant$ is backward-linear.

A history is a maximal chain in $\langle W, \leqslant\rangle$.

One intended interpretation of the above considers $W$ to be the set of possible events understood as time-slices through the whole universe and $\leqslant$ to be the "earlier-possibly later" relation. Each history represents one complete way the world could unfold.

In philosophy, BT has been widely used, especially in discussions of agency and future contingents. Unfortunately, for some goals the approach is unwieldy: in any history any two events are ordered. This is not convenient if one has in mind portraying independent choices of two agents or modelling experiments which take part in spatiotemporally separated regions.

To overcome this difficulty, a natural first step is to make events "smaller"they should represent the action in bounded spatiotemporal regions or even, ideally, point events. ${ }^{1}$ If so, then histories can no longer be chains. The guiding idea of the BST approach is that histories should represent space-times.

\subsection{Branching Space-Times structures}

In the words of its creator, the goal of the BST theory is to "combine relativity and indeterminism in a rigorous theory". ${ }^{2}$ The indeterministic aspect is carried over from the BT approach, and the relativistic aspect is to be achieved by re-imagining the notion of history. However, BST is not a straightforward generalization of BT: it will become evident after the definition of a BST structure is given that not all BT structures are BST structures. 
The definition of a BST structure is significantly more complicated than that of a BT structure; it refers to the "new" notion of history and the notion of a choice point, which we will now define.

Definition 2 A $B S T$ history is a maximal upward directed set. (A set is upward directed iff for any two its elements $e_{1}, e_{2}$ it contains an element $e$ such that $e_{1} \leqslant e$ and $e_{2} \leqslant e$.)

A choice point between two histories $h_{1}$ and $h_{2}$ is a point $e$ maximal in the intersection $h_{1} \cap h_{2}$.

We say that points $e$ and $f$ are space-time related (SLR) if there is a history $h$ such that $e, f \in h$ but neither $e \leqslant f$ nor $f \leqslant e$.

In BST, we have two types of "forward branching": modal (think of a real choice, an event having two possible futures) and non-modal (e.g. emission of particles from a source). In contrast, there is no modal backward branching: every event has a fixed past. This is because events are to be thought of as tokens, not types.

Definition 3 A $B S T$ structure is a tuple $\langle W, \leqslant\rangle$, where:

- $W \neq \emptyset$

- $\leqslant$ is a partial order on $W$;

- $\leqslant$ is dense in $W$;

- $W$ has no maximal elements w.r.t. $\leqslant$;

- every lower bounded chain in $W$ has an infimum in $W$;

- every upper bounded chain in $W$ has a supremum in every history it is a subset of;

- (Prior choice principle ('PCP')) for any lower bounded chain $O \subseteq h_{1}-h_{2}$ there exists a choice point $e \in W$ for $h_{1}$ and $h_{2}$ such that $\forall e^{\prime} \in O e<e^{\prime}$.

We do not have space here to present a detailed motivation of the conditions; they mostly stem from the two ideas of histories representing space-times (for which the conditions are still not enough; see below) and events being understood as token entities.

In BST $W$ is interpreted as containing all possible point events (ideally, each event is located in a single space-time point). $\leqslant$ is to be read as the ordering of possible causal influence, frequently interpreted as the light cone ordering: $e \leqslant f$ iff $f$ is in the future light cone of $e$.

There have been two main areas of applying BST. Some researchers used the approach to model the various experiments connected with the Bell theorem. Perhaps the non-probabilistic GHZ setup proved to be the most tractable by 
means of BST, but the probabilistic setups have also been the topic of discussion among BST theorists. ${ }^{3}$ The second area to which BST has been recently applied is that of agency. ${ }^{4}$

We will soon define the so called "transition structures" of BST structures; a transition structure will be a "skeleton" of the given BST structure, containing all the important information about branching. The current project aims to explore the remarkable similarity of these transition structures to some structures found in computer science-most notably the "event structures for concurrent processes" and Chu spaces. In the process we hope to answer some open problems about BST and provide a new, spatiotemporal reading to the aforementioned structures.

First, though, a disclaimer is in order. We said above that in BST histories are to represent space-times. The definition of a BST structure is, unfortunately, not enough for this. There are BST structures which cannot be provided with a useful notion of a space-time point. ${ }^{5}$ Still, there is a class of BST structures, called "Minkowskian Branching Structures" (MBSs), in which all histories are isomorphic to the Minkowski space-time. ${ }^{6}$ Since relativistic aspects are not relevant for the task at hand, we will assume that all considered BST structures are MBSs and thus we can think of histories as of copies of the Minkowski space-time.

For example, the following picture represents a BST structure with four histories and two binary choice points:
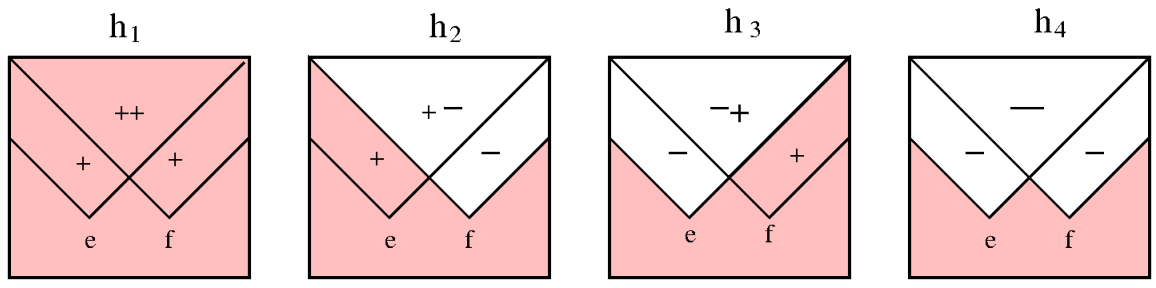

where the shaded sections denote the regions of intersection of the particular history with $h_{1}$.

\subsection{Where the action is: transitions}

BST structures are "big" in the sense that they encompass whole space-times; it would seem however that in some cases - like the simple 4-history structure above - it should be possible to distill the information about "what really happens" in the model and store it in some discrete format. According to the way of thinking about what happens in BST structures established in the literature any action goes on at a choice point, in which an "immediate possibility" is chosen. What happens in a BST structure are "transitions". In the following assume $H i s t$ to be the set of all histories in the model and $H_{(e)}$ to be the set of histories containing the point $e$. 
Definition 4 We say that histories $h_{1}$ and $h_{2}$ do not divide at $e\left(h_{1} \equiv_{e} h_{2}\right)$ iff $\exists_{e^{*}>e} e^{*} \in h_{1} \cap h_{2}$ (the two histories share a point above $e$ ).

Since $\equiv_{e}$ is an equivalence relation on $H_{(e)}$, we can, for any $e$, partition the $H_{(e)}$ into the equivalence classes of $\equiv_{e}$, to be thought of as "immediate possibilities (open) at $e^{\prime \prime}$.

Definition 5 A transition is a pair

$\langle$ a choice point $e$, an immediate possibility open at $e\rangle$.

We will assume the usual practice of denoting transitions using arrows. For example, in the picture above, we can label the two immediate possibilities open at the binary choice point $e$ as " $+e$ " and "- $e$ " and consider two transitions $e \longmapsto+_{e}$ and $e \longmapsto-{ }_{e}$.

The set $T R(O W)$ of all transitions in a BST structure $O W^{7}$ can be given a natural partial order $\leqslant_{T}$ by taking the reflexive closure of $<_{T}$, defined as follows, for $t_{i}=e_{i} \longmapsto H_{i}$ :

$$
t_{i}<_{T} t_{j} \text { iff } e_{i}<e_{j} \text { and } H_{\left(e_{j}\right)} \subseteq H_{i} .
$$

One could expect that, given a BST structure, its transition structure together with the information about the location of branching should be enough to store all the "relevant" information about the initial structure in a discrete format; relevant in the sense that (a structure isomorphic to) the initial structure should be recoverable from the discrete data. It turns out that this is not true: as we will see in the next subsection, there are different BST structures which share the same transition structure.

\subsection{Consistency and modal unsaturation}

We will call a set of transitions "consistent" if, colloquially, they can all happen together, or - in other words - there is a history in which all of them are realised. Formally, a set of transitions $\left\{t_{i} \longmapsto H_{i} \mid i \in I\right\}$ is consistent if $\exists h \in$ Hist $h \in \bigcap_{i \in I} H_{i}$; it is inconsistent otherwise. Two different transitions from the same event $e$ are called blatantly inconsistent. Histories correspond to maximal consistent sets of transitions.

We now come to an interesting feature of some BST structures (in fact, it may be the feature which makes them useful for modelling quantum experiments): that of modal unsaturation. (Usually called "funny business" in the literature; we will sometimes also use this term.) The essence is this: it might happen that some "combinatorically possible" history is missing from the structure - for example we can have two SLR binary choice points $e$ and $f$, four transitions $e \longmapsto+_{e}, e \longmapsto--_{e}, f \succ+_{f}$ and $f r_{f}$, such that the only two pairs of consistent transitions are $\left\{e \longmapsto+_{e}, f \longmapsto+_{f}\right\}$ and $\left\{e \longmapsto--_{e}, f \longmapsto--_{f}\right\}$ ! In other words, if you deleted the histories $h_{2}$ and $h_{3}$ from the structure depicted 
on p. 4, you would end up with a perfectly fine BST structure, with exactly the same transition structure.

To sum up: the goal is to give a discrete representation of a given BST structure $O W$ using the structure of its transitions, $T R(O W)$, and the information about the location of branching. An isomorphic BST structure should be recoverable from the discrete representation. This goal has been achieved by Thomas Müller in $2010,{ }^{8}$ but only for modally saturated BST structures. It would seem that the discrete format for BST structure representation should simply contain another "module" - apart from the transition structure and the spatiotemporal information - whose purpose would be to code which of the combinatorically possible histories are there in the structure. However, so far all attempts at providing such a coding have been inadequate. This points us to the first problem we want to put forward in the current paper:

Problem 1 Create a format for discrete representation of arbitrary BST structures, such that a structure isomorphic to the original one may be recovered on the basis of the representation. This would generalise Müller's 2010 theorem to arbitrary BST structures.

Again, in this short paper we cannot introduce Müller's approach. ${ }^{9}$ Let us just note that we believe the above problem belongs to an interesting type: it looks really easy, ${ }^{10}$ but has so far proven to be resistant to the attempts at solving it. Perhaps this points out that we still do not understand some basic facts about transitions structures in BST.

To recapitulate, in the move from a BST structures to its transition structure there is a loss of information which prevents the move in the opposite direction: two non-isomorphic BST structures may have identical transition structures. Solving problem 1 requires a rigorous description of that loss. It is possible that using the tools from modal logic, and more specifically the notion of bisimulation, will be fruitful in that regard. ${ }^{11}$

Suppose a propositional language is given with a single modal operator. Each BST structure $\langle W, \leqslant\rangle$ may be regarded as a modal frame, with $\leqslant$ being the accessibility relation. A $B S T$ model is a triple $\langle W, \leqslant, V\rangle$, where $\langle W, \leqslant\rangle$ is a BST structure, and $V$ is a valuation on $W$ which in a sense does justice to the splitting inherent in the structure. ${ }^{12}$ The following defines the notion of a bisimulation between two BST models: ${ }^{13}$

Definition 6 Suppose $\mathcal{M}=\langle W, \leqslant, V\rangle$ and $\mathcal{M}^{\prime}=\left\langle W^{\prime}, \leqslant^{\prime}, V^{\prime}\right\rangle$ are two BST models. A non-empty relation $Z \subseteq W \times W^{\prime}$ is a bisimulation between $\mathcal{M}$ and $\mathcal{M}^{\prime}$ when it satisfies the following three conditions, for any $w \in W, w^{\prime} \in W^{\prime}$ :

- if $w Z w^{\prime}$, then $w$ and $w^{\prime}$ satisfy the same propositional letters;

- for any $v \in W$, if $w Z w^{\prime}$ and $w \leqslant v$, then there exists a $v^{\prime} \in W^{\prime}$ such that $v Z v^{\prime}$ and $w^{\prime} \leqslant v^{\prime}$

- for any $v^{\prime} \in W^{\prime}$, if $w Z w^{\prime}$ and $w^{\prime} \leqslant^{\prime} v^{\prime}$, then there exists a $v \in W$ such that $v Z v^{\prime}$ and $w \leqslant v$. 
$Z$ is called a total bisimulation if for any $w \in W$ there exists a $w^{\prime} \in W^{\prime}$ such that $w Z w^{\prime}$, and for any $w^{\prime} \in W^{\prime}$ there exists a $w \in W$ such that $w Z w^{\prime}$.

Remember that in this paper all points in BST structures have space-time locations and all histories in all BST structures are isomorphic to the Minkowski space-time.

Conjecture 1 Suppose $O W=\langle W, \leqslant\rangle$ and $O W^{\prime}=\left\langle W^{\prime}, \leqslant^{\prime}\right\rangle$ are two $B S T$ structures. Then $T R(O W)=T R\left(O W^{\prime}\right)$ iff there exist two BST models $\mathcal{M}=$ $\langle W, \leqslant, V\rangle$ and $\mathcal{M}^{\prime}=\left\langle W^{\prime}, \leqslant^{\prime}, V^{\prime}\right\rangle$ with a total bisimulation $Z$ between them such that for any $w \in W, w^{\prime} \in W^{\prime}$, if $w Z w^{\prime}$, then $w$ and $w^{\prime}$ have the same space-time location.

Solving problem 1 would go a long way towards establishing the connection between BST and computer science. We aim to show this in the next two sections. Computer science contains numerous approaches to concurrent behaviour. ${ }^{14}$ Of these we choose two: event structures and Chu spaces.

\section{Event structures (for concurrent processes)}

We will follow the presentation of the event structures framework from a paper by Varacca, Völzer and Winskel. ${ }^{15}$

Definition 7 An event structure (ES) is a triple $\mathcal{E}=\langle E, \leqslant, \#\rangle$ such that:

- $E$ is countable;

- $\leqslant$ is a backward-finite partial order on $E$;

- \# is an irreflexive and symmetric relation (the conflict relation) such that for every $e_{1}, e_{2}, e_{3} \in E$, if $e_{1} \leqslant e_{2}$ and $e_{1} \# e_{3}$, then $e_{2} \# e_{3}$.

The authors speak of $E$ as the set of events and of $\leqslant$ as the causal order.

Notice the similarity of the conflict relation from ES with the modal branching of BST: if two events are in conflict, their descendants are also in conflict; if two BST histories branch, they never converge again.

If not for the requirement of backward-finitude, all BST structures $\langle O W, \leqslant\rangle$ and also their transition structures $\left\langle T R(O W), \leqslant_{T}\right\rangle$ would be ESs:

- for two BST events $e, f$, we put $e \# f$ iff $\neg \exists_{h \in H i s t} e, f \in h$;

- for two transitions $t_{1}, t_{2}$ we put $t_{1} \# t_{2}$ iff $\left\{t_{1}, t_{2}\right\}$ is inconsistent.

The BST structures we are dealing with are all uncountable (even if they have just a single history, it is isomorphic to the Minkowski space-time), but their transition structures may well be countable and backward finite. In general, it is clear that any BST structure $O W$ whose $T R(O W)$ is countable and backward finite determines an ES. This prompts a question about the other direction. 
Problem 2 What are the conditions for an event structure to be isomorphic to a transition structure $T R(O W)$ for some BST structure $O W$ ?

Notice that definitely not all ESs are "suitable", because in BST we do not have "trivial" transitions, i.e. transitions whose first element would be an event which is not a choice point. And so consider an ES consisting of just a single event with the empty conflict relation: it lacks a natural BST reading, since there is no BST structure with just a single transition. ${ }^{16}$

\subsection{Funny business causes confusion}

It is interesting that the framework of event structures has a notion, called confusion, which seems to be similar to the BST notion of modal unsaturation (or "funny business").

In the following assume that a configuration of an ES $\mathcal{E}$ is a conflict-free downward closed subset of $E$. (And so maximal configurations in ESs correspond to the BST histories.) Also, define $[e]:=\{x \mid x \leqslant e\}$ and $[e):=[e] \backslash\{e\}$.

Definition 8 Events $e_{1}$ and $e_{2}$ are in immediate conflict $\left(e_{1} \#_{\mu} e_{2}\right)$ when $e_{1} \# e_{2}$ and both $\left[e_{1}\right) \cup\left[e_{2}\right]$ and $\left[e_{1}\right] \cup\left[e_{2}\right)$ are configurations.

A set of events $C$ is a partial cell if for any distinct $e, e^{\prime} \in C e \#_{\mu} e^{\prime}$ and $[e)=\left[e^{\prime}\right)$. A cell is a maximal partial cell.

We hope the Reader will share the intuition that the ES notion of immediate conflict is similar in spirit to the BST notion of blatant inconsistency. It would also seem that all transitions from a single choice point to its immediate possibilities should, after the move from BST to ES, form a cell. This however may not be true if the BST structure exhibits modal unsaturation. The following table depicts the simple example of two BST structures having two binary choice points (and so the corresponding event structures are just four-element anti-chains), such that the first is exactly the modally saturated one depicted on p. 4, and the second one lacks one history. Notice that since the ESs are anti-chains, immediate conflict is just the "regular" conflict in this example.

In the first case the conflict relation joins only the elements corresponding to the transitions which are blatantly inconsistent in BST, and so the cells in the ES correspond to the sets of all transitions from a given choice point in the BST structure.

\section{BST:}

\begin{tabular}{l}
\hline \hline Two SLR binary choice points \\
$e, f ; 4$ transitions \\
\hline modal saturation \\
\hline modal unsaturation: \\
the " ++ " history excluded
\end{tabular}

ES:

\begin{tabular}{l}
\hline \hline the anti-chain $\left\{e^{+}, e^{-}, f^{+}, f^{-}\right\}$ \\
\hline$e^{+} \# e^{-}, f^{+} \# f^{-}$ \\
2 cells: $\left\{e^{+}, e^{-}\right\},\left\{f^{+}, f^{-}\right\}$. \\
\hline 3 cells: $\left\{e^{+}, e^{-}\right\},\left\{f^{+}, f^{-}\right\}$, \\
and $\left\{e^{+}, f^{+}\right\}$.
\end{tabular}


However, when modal unsaturation enters the picture, the cells are no longer disjoint and closed under $\#_{\mu}$, thus losing their intuitive BST interpretation! It turns out that the ES framework has a notion pertaining to such cases:

Definition 9 An ES is confusion free if all its cells are closed under immediate conflict.

The above discussion prompts the following conjecture:

Conjecture 2 Suppose $O W$ is a BST structure such that $T R(O W)$ is countable and backward finite. Then $O W$ is modally saturated iff $T R(O W)$ understood as an ES is confusion-free.

If the conjecture is true, we will have a mathematical link between intuitively different concepts of "lack of a combinatorically possible option" (BST) and "weird choice structure" (ES).

To conclude this section: while it is easy how (if the cardinality requirements are met) to generate an ES isomorphic to the transition structure of a given BST structure, we are searching for a general method of proceeding in the other direction.

\section{Chu spaces}

The last framework to be considered is that of Chu spaces, for which our main reference is a paper by Pratt. ${ }^{17}$ The Chu spaces are objects which are simple to define, but possess some great mathematical properties. To quote Pratt: ${ }^{18}$ they form a "remarkably well-endowed category, concrete and coconcrete, self-dual, bicomplete, and symmetric monoidal closed", serving as "a process algebra representation of linear logic", "unifying relational structures, topology, and duality into a unified framework", providing a "process interpretation of wavefunctions" and (!) " a solution to Descartes' problem of the mechanism by which the mind interacts with the body" (emphasis added). Despite all this richness, for our goals it will suffice to think of Chu spaces as two-dimensional matrices. ${ }^{19}$

Definition 10 A Chu space over a set $K$ is an $A \times X$ matrix whose elements are drawn from $K$.

In all the Chu spaces we will consider the set $K$ is equal to $\{0,1\}$.

Despite the apparent simplicity, the framework carries with itself robust interpretations of rows and columns of the matrices. If we view a given space by rows, then $A$ is the "carrier of structure";"20 a row labeled $e$ is the complete description of the element $e$. If we view it by columns, $A$ is a set of "locations" (variables) and each column is a permitted assignment of values from $K$ to them. $^{21}$ In our case rows will be labeled by transitions, and the "permitted assignments" will correspond to the characteristic functions of consistent sets of transitions (and the empty set). 
Formally, the following is a method of representing BST structures by means of Chu spaces (notice the lack of cardinality requirements):

- there is a $1-1$ correspondence between the rows of the space and the transitions of the BST structures, which serve as labels for the rows;

- each column codes a possible past of an event in the BST structure, with "1" at all and only the rows whose corresponding transitions already happened from the perspective of the given point.

\subsection{BST structures and "their" Chu spaces}

The above will hopefully be made clearer by a few examples. We will always omit column labels. The Chu space corresponding to a BST structure with a single binary choice point (and so two transitions, labelled $e^{+}$and $e^{-}$) is the following:

\begin{tabular}{|l||l|l|l|}
\hline$e^{+}$ & 0 & 1 & 0 \\
\hline$e^{-}$ & 0 & 0 & 1 \\
\hline
\end{tabular}

There are three columns because each event in the BST structure has one of the three possible pasts: it may be so that from its perspective $e^{+}$already happened, or that $e^{-}$did, or none of those happened (yet). Since it is impossible for an event to have both $e^{+}$and $e^{-}$in its past, as the two transitions are blatantly inconsistent, there is no column with two 1's.

A modally saturated BST structure with two binary choice points $e$ and $f$ (e.g. the one depicted on p. 4) gives rise to the following Chu space:

\begin{tabular}{|l||l|l|l|l|l||l|l|l|l|}
\hline$e^{+}$ & 0 & 1 & 0 & 0 & 0 & 1 & 1 & 0 & 0 \\
\hline$e^{-}$ & 0 & 0 & 1 & 0 & 0 & 0 & 0 & 1 & 1 \\
\hline$f^{+}$ & 0 & 0 & 0 & 1 & 0 & 1 & 0 & 1 & 0 \\
\hline$f^{-}$ & 0 & 0 & 0 & 0 & 1 & 0 & 1 & 0 & 1 \\
\hline
\end{tabular}

The double line after the fifth column serves just to mark the point behind which each the columns determine which combinatorically possible histories are there in the model. Since the structure is modally saturated, all 4 possible histories are there.

The simplest case of modal unsaturation, with just a single history ("++") excluded, amounts just to the deletion of one column:

\begin{tabular}{|l||c|c|c|c|c||c|c|c|}
\hline$e^{+}$ & 0 & 1 & 0 & 0 & 0 & 1 & 0 & 0 \\
\hline$e^{-}$ & 0 & 0 & 1 & 0 & 0 & 0 & 1 & 1 \\
\hline$f^{+}$ & 0 & 0 & 0 & 1 & 0 & 0 & 1 & 0 \\
\hline$f^{-}$ & 0 & 0 & 0 & 0 & 1 & 1 & 0 & 1 \\
\hline
\end{tabular}

Notice that if we deleted all the columns in which two transitions happened, we would get the following: 


\begin{tabular}{|l||c|c|c|c|c|}
\hline$e^{+}$ & 0 & 1 & 0 & 0 & 0 \\
\hline$e^{-}$ & 0 & 0 & 1 & 0 & 0 \\
\hline$f^{+}$ & 0 & 0 & 0 & 1 & 0 \\
\hline$f^{-}$ & 0 & 0 & 0 & 0 & 1 \\
\hline
\end{tabular}

which naturally represents a BST structure with just a single choice point with 4 immediate possibilities.

The last example should suggest that the relationship between BST structures and $\mathrm{Chu}$ spaces is not entirely straightforward. To reinforce this point, notice that not all Chu spaces over $\{0,1\}$ have a natural BST reading. Consider the following:

\begin{tabular}{|l||l|l|l|l|l||l|}
\hline$e^{+}$ & 0 & 1 & 0 & 0 & 0 & 1 \\
\hline$e^{-}$ & 0 & 0 & 1 & 0 & 0 & 0 \\
\hline$f^{+}$ & 0 & 0 & 0 & 1 & 0 & 0 \\
\hline$f^{-}$ & 0 & 0 & 0 & 0 & 1 & 1 \\
\hline
\end{tabular}

It cannot represent a structure with a single choice point, because the last column indicates that it is possible to have two transitions in the past. But there seems to be no way of looking at this space as representing a BST structure with two or any other number of choice points.

Notice that the above examples show that sometimes, starting from a Chu space representing a modally saturated BST structure, one can, just by deleting columns which seemingly correspond to combinatorically possible histories, introduce modal unsaturation, then lose the natural BST reading altogether, and eventually end up with a space representing a BST structure with a different number of choice points. Contrast this with the process of removing histories from the modally saturated BST structure depicted on p. 4: if we remove one history (say $h_{2}$ ), we introduce modal unsaturation, if we remove two histories (say $h_{2}$ and $h_{3}$; notice that not all choices are permissible), we again have a structure with modal unsaturation, if we remove three histories we get a structure with no choice points at all, and if we remove all four histories we end up with the empty set. Perhaps a general theorem on the relationship between BST structures and $\mathrm{Chu}$ spaces requires a deeper understanding of the connection between columns representing the "latest possible pasts" (i.e. the right-most columns in our examples) and the combinatorically possible histories in BST structures.

We can, however, provide a procedure which given a Chu space $A^{\prime} \times X^{\prime}$ over $\{0,1\}$ (with $A^{\prime}$ countable) creates an event structure $\langle E, \leqslant, \#\rangle$ (whenever $\leqslant$ turns out to be backward-finite):

1. Delete any repeated rows from $A^{\prime}$ and columns from $X^{\prime}$ (save for a single copy in each case), arriving at $A$ and $X$.

2. Set $E$ to be $A$.

3. For the ordering $\leqslant$, take the bit-wise ordering of rows, given by the inverse of the "left residual" of $A \times X$ and itself: namely, the set of pairs $\langle b, a\rangle$ of 
elements of $A$ such that for any column $x \in X$, if there's a 1 at row $a$ and column $x$, then there is a 1 at row $b$ and column $x$ (in such a case we want to say that $b \leqslant a$ ).

4. Set $e \# f$ for any and all $e, f \in A$ such that no column contains 1's at both rows $e$ and $f$.

Were we able to prove the theorem about discrete representations of BST structures in full generality (see Problem 1), we could move all the way from Chu spaces, via event structures, to BST structures. As things stand, the known method $^{22}$ of constructing a BST structure on the basis of a given transition structure always generates a modally saturated BST structure.

We are left with a similar problem as in the case of event structures:

Problem 3 What are the conditions for a Chu space over $\{0,1\}$ to generate a transition structure $T R(O W)$ for some BST structure $O W$ ?

\section{Conclusion}

In this paper we put forward two conjectures and three problems regarding the relationship of BST structures, event structures for concurrent processing and $\mathrm{Chu}$ spaces. It seems to be relatively easy to generate the latter objects given BST structures (preserving what we believe to be important: the shape of the transition structure), and more difficult to move in the other direction.

We hope that in the process of investigating these problems we will gain some insight into the relationship between concepts from seemingly unrelated fields of philosophy and computer science, between which nonetheless there definitely seems to be a mathematical connection: as an example, take the notion of modal unsaturation (BST) and confusion (ES), the topic of section 3.1.

The investigation so far suggests that there is much to gain in this for BST theorists - using the tools from computer science may offer a new look at some BST problems and provide a better understanding of transition structures. Still, perhaps some computer scientists will also be interested in spatiotemporal readings of their structures.

\section{Acknowledgements}

We would like to thank the Reviewer for fruitful comments and Thomas Müller for suggesting the topic in the first place and sharing his thoughts on the key issues of the paper. Our thanks also go to Vincent van Oostrom for a brainstorming discussion. The research was supported by the MNiSW grant no. 668/N-RNP-ESF $/ 2010 / 0$

Author's address:

Institute of Philosophy

Jagiellonian University 
Grodzka 52

31-044 Kraków

Poland

leszek.wronski@uj.edu.pl 


\section{Notes}

${ }^{1}$ For the discussion of point events see the founding paper for the BST theory: Nuel Belnap, 1992, "Branching Space-Time", in: Synthese 92, 3, pp. 385-434.

${ }^{2}$ Ibid., p. 385.

${ }^{3}$ See e.g. Nuel Belnap and László Szabó, 1992, "Branching Space-Time Analysis of the GHZ Theorem", in: Foundations of Physics 26, 8, pp. 989-1002; and Tomasz Placek, 2010, "On Propensity-Frequentist Models for Stochastic Phenomena with Applications to Bell's Theorem", in: Tadeusz Czarnecki, Katarzyna Kijania-Placek, Olga Poller and Jan Woleński (Eds.), The Analytical Way, London: College Publications, pp. 105-140.

${ }^{4}$ See e.g. Nuel Belnap, 2011, "Prolegomenon to Norms in Branching Space-Times", in: Journal of Applied Logic 9, pp. 83-94.

${ }^{5}$ See Thomas Müller, 2005, "Probability Theory and Causation: A Branching Space-Times Analysis", in: British Journal for the Philosophy of Science 56, 3, pp. 487-520.

${ }^{6}$ See Thomas Müller, 2002, "Branching Space-Time, Modal Logic and the Counterfactual Conditional", in: Tomasz Placek and Jeremy Butterfield (Eds.), Non-locality and Modality, Drodrecht:Kluwer, pp. 273-291 and Leszek Wroński and Tomasz Placek, 2009, "On Minkowskian Branching Structures", in: Studies in History and Philosophy of Science Part B: Studies in History and Philosophy of Modern Physics 40, 3, pp. 251-258.

${ }^{7}$ This is an abbreviation dating back to the 1992 paper by Belnap (op. cit.), denoting "Our World".

${ }^{8}$ See Thomas Müller, 2010, "Towards a Theory of Limited Indeterminism in Branching Space-Times", in: Journal of Philosophical Logic 39, pp. 395-423.

${ }^{9}$ Building the formal machinery needed for this required many pages in ibid.

${ }^{10}$ This opinion is based on conversations with people accustomed with the BST framework.

${ }^{11}$ We are very grateful to the Reviewer for suggesting this.

${ }^{12}$ For details, see e.g. Tomasz Placek and Nuel Belnap, 2010, "Indeterminism is a modal notion: branching spacetimes and Earman's pruning", in: Synthese, DOI 10.1007/s11229-0109846-8.

${ }^{13}$ For a description of the notion in the general context of modal logic, see e.g. Patrick Blackburn, Maarten de Rijke and Yde Venema, 2001, Modal logic, Cambridge University Press.

${ }^{14}$ For an overview see Vaughan Pratt, 2003, "Transition and Cancellation in Concurrency and Branching Time", in: Mathematical Structures in Computer Science 13, 4, pp. 485-529.

${ }^{15}$ Daniele Varacca, Hagen Völzer, and Glynn Winskel, 2006, "Probabilistic Event Structures and Domains", in: Theoretical Computer Science 358, 2-3, pp. 173-199.

${ }^{16}$ As a side-note, the following is a problem stated only once the connection between BST and ES has been noticed, but which relates to the current lack of deeper understanding of some fundamental aspects of BST: what are the sufficient and necessary conditions for a set of transitions to be the set of all transitions for some BST history?

${ }^{17}$ Vaughan Pratt, 1995, "Chu Spaces and Their Interpretation as Concurrent Objects", in: Computer Science Today 1000, pp. 392-405 (2005 version from the Author's homepage, http://boole.stanford.edu/pub/chuconc.pdf ).

${ }^{18}$ Ibid., p. 3

${ }^{19}$ The mathematical value of Chu spaces seems to stem from the so called "Chu transforms", not introduced in this paper.

${ }^{20}$ Ibid.

${ }^{21}$ Perhaps the Reader will find the following quote illuminating: "The rows present the physical, concrete, conjunctive, or yang aspects of the space, while the columns present the mental, coconcrete, disjunctive, or yin aspects" (ibid., p. 4).

${ }^{22}$ See Müller, "Towards a Theory of Limited Indeterminism in Branching Space-Times", op. cit. 